San Jose State University

SJSU ScholarWorks

Master's Projects

Master's Theses and Graduate Research

$1-1-1997$

\title{
Evaluation of The Effectiveness of a Direct Observed Therapy Program With Vietnamese Tuberculosis Patients
}

Jacqueline T. Mac

San Jose State University

Follow this and additional works at: https://scholarworks.sjsu.edu/etd_projects

Part of the Public Health and Community Nursing Commons

\section{Recommended Citation}

Mac, Jacqueline T., "Evaluation of The Effectiveness of a Direct Observed Therapy Program With

Vietnamese Tuberculosis Patients" (1997). Master's Projects. 869.

DOI: https://doi.org/10.31979/etd.uacr-6efe

https://scholarworks.sjsu.edu/etd_projects/869

This Master's Project is brought to you for free and open access by the Master's Theses and Graduate Research at SJSU ScholarWorks. It has been accepted for inclusion in Master's Projects by an authorized administrator of SJSU ScholarWorks. For more information, please contact scholarworks@sjsu.edu. 
Running Head: DIRECT OBSERVED THERAPY PROGRAM

Evaluation of The Effectiveness of a Direct Observed Therapy Program With Vietnamese Tuberculosis Patients

\author{
Jacqueline T. Mac, RN, PHN, MSN
}

Santa Clara County Public Health Department

Ann Doordan, PhD, RN

Professor, School of Nursing

San Jose State University

Catherine A. Carr, CNM, DrPH

Program Director Midwifery Education Program

Education Programs Associates (EPA)/San Jose State University

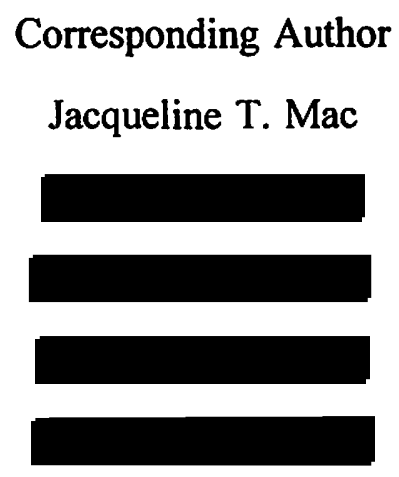




\begin{abstract}
Tuberculosis (TB) has long been recognized as a major public health problem. The rate of TB is high in immigrants and the frequency of drug resistance is increasing. A major reason for the development of resistant infections as well as relapse is poor adherence to TB treatment. In response to this problem, direct observed therapy (DOT) was introduced to the TB program in Santa Clara County in 1993. The purpose of this study is to compare the completion rates, relapse rates, and sputum conversion rates between a DOT group and a non-DOT group of Vietnamese TB patients. A chart review was completed with a convenience sample of 25 records of DOT patients and 25 records of non-DOT patients. Frequencies and percentages were used to analyze the completion rates and the relapse rates. The results show that the completion of therapy rate was $16 \%$ higher in the DOT group and the relapse rate was $8 \%$ lower. A t-test indicated that the sputum conversion rate was significantly more rapid in the DOT group than in the non-DOT group $(p<.05)$. It appears that Vietnamese TB patients benefit from the DOT program.
\end{abstract}


Evaluation of The Effectiveness of a Direct Observed Therapy Program With Vietnamese Tuberculosis Patients Tuberculosis (TB) has regained the attention of the medical community over the last decade as a major public health concern. Although TB affects many segments of society in the United States (U.S.), it is of special concern for people from endemic countries. According to the Centers for Disease Control and Prevention (CDC) (1994), people from Vietnam, Mexico, Philippines, China, Haiti, India, and Korea account for roughly two-thirds of the TB cases among foreign-born people in the U.S. In 1994, of the 7,627 foreign born residents with TB, 869 (11.4\%) were from Vietnam (CDC, 1994). In California, the rate of TB in Santa Clara County (SCC) has steadily increased in recent years. In 1996, with a rate of 18.2 TB cases per 100,000 persons, the county is far from meeting the national Year 2000 objective of 3.5 cases per 100,000 . In 1995 , SCC had the $7^{\text {th }}$ highest rate of TB among reporting jurisdictions in Califormia. By 1996, the county ranked $5^{\text {th }}$, with a TB rate higher than California (18.2 vs. 12.17). Among 296 TB cases reported by ethnicity in SCC in 1996, the Vietnamese ranked number one, with 103 cases (35\%) (Public Health Report, 1997).

In response to the rising incidence of $\mathrm{TB}$, the County Health Department began a program of direct observed therapy (DOT) in 1993 (California TB Control Report, 1993). DOT is a procedure in which health care workers watch or assist TB patients as they take their medication. At the same time, the health care worker is available to answer the patient's questions about TB or the TB therapy, and to help resolve any problems the patient may be having with the disease or treatment (Myers, 1996). DOT may be either clinic or field-based in 
the home, school, workplace, shelter, park, or other convenient place for patients. If a patient fails to keep an appointment for DOT, the health care worker begins to look for him or her (Myers, 1996). In SCC, public and private primary care providers may refer their TB patients to the DOT program or keep them in self-administered therapy.

\section{Purpose}

The purpose of this study is to examine the effects of a DOT program as measured by client outcomes using an ex-post facto correlation research design to analyze the relationship between the completion-of-therapy rates, relapse rates, and sputum conversion rates for a DOT group and a non-DOT group of Vietnamese TB patients.

Hypotheses

Three hypotheses consistent with outcomes pertinent to this client population and the purpose of the study were tested.

Hypothesis 1. Completion-of-therapy rates will be higher in the DOT group than in the non-DOT group.

Hypothesis 2. Relapse rates will be lower in the DOT group than in the non-DOT group.

Hypothesis 3. Sputum conversion (from positive to negative) will occur more rapidly in the DOT group than in the non-DOT group.

Background and Literature Review

Although effective drugs exist for treating TB, it is difficult to ensure completion of treatment. Cultural factors, such as misconceptions about appropriate treatment methods, in combination with other barriers, may adversely affect adherence and lead to improper uses of TB medication 
(Sumatojo, 1993). Poor adherence to treatment may contribute to the emergence of drug-resistant bacilli (Van-Si, 1992, p. 24). Some patients may not fully understand their condition, comprehend its significance, or be aware of appropriate treatment (p. 24). Differences between patients and providers' understandings of illnesses can interfere with adherence to treatment (Cohen, 1994). Patients may be reluctant to seek treatment, might not take TB medication as prescribed, and may have difficulty understanding explanations offered by health care personnel due to misconceptions about TB, communication barriers, or fear of being socially stigmatized. Previous research has shown that misconceptions about TB and other barriers to adherence are widespread in many different groups in the U.S., including the Vietnamese population (Carey, Oxtoby, Nguyen, Huynh, Morgan, \& Jeffery, 1997).

Vietnamese have traditionally dealt with illness through self-care and self-medication. When illness does occur, they typically practice self-care: coining, cupping, pinching, or using herbal medicines before seeking professional help (Mueke, 1990). In addition, self-medication is the typical medical behavior in Vietnam (Mueke, 1990). In Vietnam, a prescription to purchase medicine at a drug store is not needed. This may partially explain the increasing resistance of TB bacillus to several commonly used antibiotics (Nguyen, 1989). Vietnamese tend to define their health problem in terms of physical symptoms and to seek symptomatic treatment. When symptoms are relieved, or "cured," they are likely to discontinue the treatment (Hoang, 1991). Additionally, most Vietnamese believe that Western medicines are "hot," too strong, or are inappropriate for the Vietnamese. A typical way to avoid the problem of "hot" medicine is to reduce the recommended dosage or to stop 
taking the medicine prematurely (Waxler-Morrison, 1990, p. 196). It is difficult to convince Vietnamese patients that long-term medical treatment (such as TB treatment) is beneficial, particularly when there are no apparent symptoms. The practice of reduced dosage and shortened treatment have contributed to antituberculosis drug-resistance problems in the U.S. (Hoang, 1991). Based on the California TB Control Report 1992, an estimated 590 (11\%) of the 5,273 total TB patients reported in 1991 did not complete appropriate treatment and had an increased risk of developing drug resistance and transmitting drugresistant strains to others.

Keane (1995) conducted a study of TB prevalence in Vietnamese emigrants. The study aimed to describe the International Organization for Migration (IOM) TB screening and treatment program in Ho Chi Minh City (Vietnam) and to review the prevalence of TB and treatment outcomes in a cohort of Vietnamese emigrants and refugees prior to their departure (from November 1, 1992 to June 1, 1993). The report confirms the efficacy of short course (6 month) chemotherapy coupled with DOT treatment for 265 TB patients, in the context of one of the largest screening programs for prospective emigrants.

In Tarrant County, Texas, a program of universal DOT for TB (all TB patients were enrolled in the DOT program) was initiated in November 1986 in response to the development of drug-resistant infection and increasing relapse rates. A study was conducted to evaluate the effect of the program on the rates of primary and acquired drug resistance and relapse among patients with TB (Weis, Slocum, Blais, King, Lunn, Matney, Gomes, \& Foresman, 1994). The authors analyzed records for all patients with positive cultures for 
mycobacterium TB in Tarrant County from January 1, 1980 through December 31, 1992. Through October 1986, patients received a traditional, unsupervised drug treatment. Beginning in November 1986, nearly all patients received therapy under direct observation by health care personnel. A total of 407 cases of TB in which patients received traditional treatment for TB (January 1980 through October 1986) were compared with 581 cases of TB in which therapy was directly observed (November 1986 through December 1992). Despite higher rates of intravenous drug use and homelessness and an increasing incidence of TB during this 13-year period, the frequency of primary drug resistance decreased from $13.0 \%$ to $6.7 \%$ after the introduction of DOT, and the frequency of acquired resistance declined from $14.0 \%$ to $2.1 \%$. The relapse rate decreased from $20.9 \%$ to $5.5 \%$ and the number of relapses with multidrugresistant organisms decreased from 25 to 5 . The study indicated that the administration of therapy for TB infection under direct observation leads to significant reductions in the frequency of primary drug resistance, acquired drug resistance, and relapse.

Community based DOT for TB control was examined by Chaulk, Moore-Rice, Rizzo, Chaisson, O'Brien, and Jacobs (1995) in Baltimore, MD. Comparisons were done with prior rates and with five other cities that had high rates of TB. Results indicated that since 1981, Baltimore experienced the greatest decline in TB incidence: 35.6 cases per 100,000 population in 1981 and 17.2 cases per 100,000 population in 1992 , a $51.7 \%$ reduction. The city fell from the $6^{\text {th }}$ highest rate for TB nationally in 1981 to $28^{\text {th }}$ in 1992 . Since 1985 , TB incidence increased $35.3 \%$ in a five-city cohort, but declined $29.5 \%$ in Baltimore. From 1986 through 1992, Baltimore's DOT-managed cases had the 
highest sputum conversion rates from positive to negative at 3 months and the highest completion rates for standard anti-TB therapy when compared with the five cities. Disease relapse rates remained low, even among HIV-infected patients. The authors concluded that Baltimore experienced a substantial decline in TB following implementation of community-based DOT, despite high rates of medicosocial risk factors. DOT also facilitated high treatment completion rates and bacteriologic evidence of cure.

The cost of DOT has been cited as a reason for not implementing DOT programs widely (Weis et al., 1994). The average cost of a non-DOT TB case with 6-month TB treatment is $\$ 12,672$, compared to $\$ 13,515$ for 6-month TB treatment of a DOT TB case (Moore, Chaulk, Griffiths, Cavalcante, \& Chaisson, 1996). Although this is an increase of about $\$ 1,000$ over the baseline direct cost of DOT, it compares favorably to the average cost of treating multidrug-resistant TB; $\$ 230,400$, with a range from $\$ 100,000$ to $\$ 500,000$ (Moore et al., 1996).

Other studies find similar efficacy rates with DOT programs (Schaaf, 1996; Nitta, 1996; Houston \& Fanning, 1994; McDonald, Menon, \& Reichman, 1982). All recommend that the high cost of treating multi-drug resistant TB patients and the danger of spreading resistant organisms make prevention increasingly important. More emphasis should be placed on DOT to cure newly diagnosed infectious drug-sensitive TB patients.

Nonadherence to medication regimens has historically been among the most challenging problems in TB management. According to Moore et al. (1996), a variety of factors are known to influence adherence, including the complexity and duration of treatment regimens, drug side-effects, cost, and 
access to health care. Factors, such as age, gender, race, level of education, and substance abuse that influence adherence to TB treatment were studied by Sumatojo (1993). Studies were conducted in different high risk populations of TB, including those with AIDS, HIV infection, homelessness, and substance abuse (Alwood, Keruly, Moore-Rice, Stanton, Chaulk, \& Chaisson, 1994; Millard, Cegielski, Wing, Silver, Cohn, \& Barbaro, 1994; Schulger, Ciotoli, Cohen, Johnson, Rom, \& Bray, 1995; Wilkinson, Davies, \& Connolly, 1996). The results show that a DOT program is capable of achieving a high degree of success in a population at high risk for noncompliance. No study about the effect of DOT on adherence to TB treatment of the Vietnamese population was found.

\section{Methodology}

\section{Sample and Setting}

This study used a structured retrospective review of 96 charts of Vietnamese TB patients who had been followed by the Public Health Department in Santa Clara County. The criteria for sample selection included Vietnamese TB patients who were: (a) 18 years or older, (b) had been diagnosed with pulmonary TB only (with positive culture for mycobacterium TB), and (c) had been initially prescribed with all four anti-TB medicines (isoniazid [INH], rifampin [RIF], pyrazinamide [PZA], and ethambutol [EMB]), and (d) had records that had been completed between December 31, 1994 and December 31, 1997. Patients with a history of AIDS, cancer, diabetes, renal disease, cardiovascular disease, leprosy, or other chronic diseases were excluded. Patients who were diagnosed with extrapulmonary or both pulmonary and extrapulmonary TB were also excluded. 


\section{Procedure}

Institutional Review Board clearance from San Jose State University and written permission from the Director of Public Health Nursing of Santa Clara County were obtained for this study prior to commencing data collection. Patient records were selected by hand and were chosen by the investigator according to common Vietnamese surnames. Records were selected according to the criteria until the sample size was achieved with 25 records of DOT patients and 25 records of non-DOT patients. The sample was one of convenience and was not randomized.

Data obtained from patient records were recorded on a 17 item chart review form designed by the investigator. Demographic data included age and gender to better describe the sample and possibly guide future research. Sensitivity or resistance was recorded for all TB medications in order to identify drug-resistant subjects. A multidrug-resistant (MDR) organism was defined as an isolate demonstrating resistance to at least two of the following drugs: isoniazid rifampin, ethambutol, or streptomycin. Primary resistance was defined as resistance to one or more drugs present on the first sensitivity test for which results were available. Acquired resistance was defined as resistance that was not present on the initial sensitivity testing but that developed during or after treatment. The date of treatment initiation, end date, and length of treatment (in months) were collected to compare the completion rate and the length of treatment of the DOT group and non-DOT group. The relapse date with or without acquired drug-resistance was noted in order to compare the relapse rates. A relapse in this study was defined as a positive culture for mycobacterium TB in a patient who had previously completed an adequate course of therapy. A 
relapse during therapy was defined as a positive culture in a patient currently under treatment who had been culture-negative for a minimum of 4 months with at least three negative cultures. A drug-resistant relapse was defined as a positive culture for drug-resistant mycobacterium TB in a patient in whom the definition of a relapse was met. The sputum conversion date (the date the sputum culture converted to negative) and length of sputum conversion (in weeks) from the date TB treatment was started to the date sputum converted to negative were recorded to compare the sputum conversion rate between the two groups. The sites where patients had medical care and the sites where therapy was observed were also recorded.

\section{Results}

The chart review was completed with the 50 patient records included in the study. Table 1 displays demographic data for the sample. Patient ages ranged from 20 to 87 years, with 29 males and 21 females. Twenty-eight patients (56\%) underwent medical care at private medical doctor (PMD) offices, 17 patients (38\%) were treated at a TB clinic, and 3 patients (6\%) had medical care at county medical clinics. Of these 50 patients, $9(18 \%)$ were resistant to anti-TB drugs.

\section{Non-DOT Group}

Among the 25 patients in the non-DOT group, 13 were males and 12 were females. Ages ranged from 20 to 87 years, with a mean age of 41.88 years. Of the 25 patients, $20(80 \%)$ were under medical care of PMDs, $2(8 \%)$ were treated at various county medical clinics, and $3(12 \%)$ were treated at the county TB clinic. Two of the patients (8\%) were resistant to anti-TB drugs and one of these patients was a multidrug-resistant TB case. 


\section{DOT Group}

There were 25 patients in the DOT group: 16 males and 9 females. Ages ranged from 21 to 81 years, with a mean age of 39.72 years. Eight patients $(32 \%)$ were under medical care of PMDs, one (4\%) was treated at the county medical clinic and $16(64 \%)$ were under medical care at the TB clinic. Seven patients $(28 \%)$ were resistant to anti-TB drugs and two of them $(8 \%)$ were multidrug-resistant TB cases.

\section{Completion of Therapy Rate}

In the non-DOT group, 21 patients (84\%) completed the TB treatment and 4 patients (16\%) were lost to follow up, compared to 25 patients (100\%) who completed the TB treatment in the DOT group. No lost-to-follow up cases were identified in the DOT group (see Table 2). Frequency and percentage were used to analyze the results. The statistics showed that the completion-of-therapy rate was $16 \%$ higher in the DOT group, supporting the first hypothesis:

completion-of-therapy rate was higher in the DOT group than in the non-DOT group.

\section{Relapse Rate}

Two patients (8\%) in the non-DOT group relapsed after completion of TB treatment; one was an acquired multidrug-resistance relapse. This compared to no relapses identified in the DOT group (Table 2). These results support the second hypothesis: the relapse rate was lower in the DOT group than in the nonDOT group.

\section{Sputum Conversion Rate}

In the non-DOT group, the length of time to sputum conversion ranged from 4 to 16 weeks with the mean of 10.29 weeks (S.D. $=2.80$ ) (Table 3). In 
the DOT group, the sputum conversion ranged from 2 to 7 weeks with the mean of 4.72 weeks (S.D. $=1.77)$ (Table 3). A t-test was used to compare the means between the two groups $t=8.200 ; d f=44 ; p<.05$ ). There was a statistically significant difference between the DOT group and the non-DOT group in the achievement of sputum conversion $(p=0.00)$. These results indicate that the DOT program has a significant impact on how rapidly the patient can accomplish sputum conversion. This supports the third hypothesis: sputum conversion occurred more rapidly in the DOT group than in the non-DOT group.

\section{Discussion}

Consistent with the findings of other investigators in different populations (Alwood et al., 1994; Chaulk et al., 1995; Houston \& Fanning, 1994; Keene, 1995; Millard et al., 1994; Nitta, 1996; Schaaf, 1996; Schulger, 1995; Weis et al., 1994; Wilkinson, Davies, \& Connolly, 1996), the results of this study indicate that a DOT program has a significant impact on the completion rate, relapse rate, and sputum conversion rate of Vietnamese TB patients. This occurs despite the higher rate of drug resistant cases in the DOT group ( $28 \%$ vs $8 \%$ ).

It appears that despite all the differences in culture, health beliefs, and health practices, the Vietnamese TB clients benefit from the DOT program. The DOT program apparently provides sufficient support and encouragement to complete TB treatment. The investigator found that the DOT patients were followed more closely by primary care providers with visits at least once a month, compared to every 2 to 3 months in the non-DOT group. In the DOT group, blood tests, pulmonary radiography, and sputum examinations were ordered as recommended by CDC guidelines to measure the progress of patients which were not regularly done in the non-DOT group. These factors may 
contribute to the successful treatment of TB patients. Treatment not only benefits the individual patient, but also benefits the public at large as infectious cases are cured and sources of infection are removed from the community. Promoting the use of effective chemotherapy and monitoring adherence to treatment are essential for treatment to be successful.

The particular nature of the setting in one public health district office and one population with a small sample size limits generalization. However, the results are promising and need to be followed up with additional studies. The rapidly growing population and increasingly diverse population of TB patients poses a real challenge to primary care providers in treating TB. Future research of diverse populations in various geographic locations and in a larger sample is needed to further identify the effectiveness of a DOT program. 


\section{References}

Alwood, K., Keruly, J., Moore-Rice, K, Stanton, B. L., Chaulk, C., \& Chaisson, R. E. (1994). Effectiveness of supervised intermittent therapy for TB in HIV-infected patients. AIDS, 8, 1103-1108.

Carey, J. W., Oxtoby, M. J., Nguyen, L. P., Huynh, V., Morgan, M., \& Jeffery, M. (1997). Tuberculosis beliefs among recent Vietnamese refugees in New York state. Public Health Report, 112, 66-72.

Centers for Disease Control and Prevention (CDC), Division of Tuberculosis Prevention. (1994). Reported tuberculosis in the United States, 1994. Atlanta: CDC.

Chaulk, C. P., Moore-Rice, K., Rizzo, R., Chaisson, R. E., O’Brien, R., \& Jacobs, R. F. (1995). Eleven years of community-based directly observed therapy for tuberculosis. Journal of American Medical Association. 274, 945951.

Cohen, M. Z. (1994). Explanatory models of diabetes: Patient practitioner variation. Social Science Medicine, 38, 59-66.

Hoang G. N. (1991). Cultural barriers to effective medical care among Indochinese patients. Annual Review of Medicine, 36, 229-230.

Houston S., \& Fanning, A. (1994). Current and potential treatment of tuberculosis. Drugs, 48, 689-708.

Keane, V. P. (1995). Prevalence of tuberculosis in Vietnamese migrants: The experience of the Orderly Departure Program. Southeast Asian Journal of Tropical Medicine of Public Health, 26, 642-647. 
McDonald R. J., Menon A. M., \& Reichman, L. B. (1982). Successful supervised ambulatory management of tuberculosis treatment failures. Annals of Internal Medicine, 96, 297-302.

Moore R. D., Chaulk, C. P., Griffiths, R., Cavalcante, S., \& Chaisson, R. E. (1996). American Journal of Respiratory Critical Care Medicine, 154, 10131019.

Myers, C. S. (1996). Eying TB therapy. The Journal for Respiratory Care Practitioners. 4, 93-74, 126.

Millard, P. S., Cegielski, J. P., Wing, S., Silver, A., Cohn, D. L., \& Barbaro, J. A. (1994). Rurality and tuberculosis incidence trends in North and South Carolina. Journal of Rural Health. 10, 226-236.

Muecke, M. (1990). Vietnamese refugees in the American health care system. American Journal of Public Health, 173, 831-838.

Nitta, A. (1996). Multidrug-resistant tuberculosis-How far are we from New York City? Western Journal of Medicine, 165, 225-226.

Nguyen, D. M. (1989). Culture shock: A review of Vietnamese culture and its concepts of health and disease. Western Journal of Medicine, 242, 819822.

Santa Clara County Health Department. (1992). California TB Control Report 1992. San Jose, CA: Author.

Santa Clara County Health Department. (1993). California TB Control Report 1993. San Jose, CA: Author.

Santa Clara County Health Department. (1997). Public Health Report 1997. San Jose, CA: Author. 
Schaaf, H. S. (1996). The 5-year outcome of multidrug-resistant tuberculosis patients in the Cape Province of South Africa. Tropical Medicine and International Health, 1, 718-722.

Schulger, N., Ciotoli, C., Cohen, D., Johnson, H., Rom, W. N., \& Bray, D. (1995). Comprehensive TB control for patients at high risk for noncompliance. American Journal of Respiratory and Critical Care Medicine. 151, 1486-1490.

Sumatojo, E. (1993). When tuberculosis treatment fails: A social behavior account of patient adherence. The American Review of Respiratory Discase. 147, 1311-1320.

Van-Si, C. (1992). Understanding Southeast Asian culture: Their cultural traits and implications in casework practice. Oak Grove, IL: Asian American United Press.

Waxler-Morrison, N. (1990). Cross-cultural caring. Vancouver: The University of British Columbia Press.

Weis, S. E., Slocum, C. C., Blais, F. X., King, B., Lunn, M., Matney, G. B., Gomes, E., \& Foresman, B. H. (1994). The effect of DOT on the rates of drug resistance and relapse in TB. The New England Journal of Medicine, 330 , 1179-1184.

Wilkinson D., Davies, G. R., \& Connolly, C. (1996). Directly observed therapy for tuberculosis in rural South Africa, 1991 through 1994. American Journal of Public Health, 86, 1094-1097. 
Table 1

Demographic Data

\begin{tabular}{|c|c|c|c|c|c|c|}
\hline & \multicolumn{2}{|c|}{$\begin{array}{l}\text { Combined Group } \\
\qquad(\mathrm{N}=50)\end{array}$} & \multicolumn{2}{|c|}{$\begin{array}{l}\text { DOT Group } \\
\qquad(n=25)\end{array}$} & \multicolumn{2}{|c|}{$\begin{array}{l}\text { Non-DOT Group } \\
\qquad(n=25)\end{array}$} \\
\hline & Frequency & Percent & Frequency & Percent & Frequency & Percent \\
\hline \multicolumn{7}{|l|}{ Gender } \\
\hline Male & 29 & 58 & 16 & 64 & 13 & 52 \\
\hline Female & 21 & 42 & 9 & 36 & 12 & 48 \\
\hline \multicolumn{7}{|l|}{ Age } \\
\hline $20-29$ & 13 & 26 & 9 & 36 & 4 & 16 \\
\hline $30-39$ & 14 & 28 & 4 & 16 & 10 & 40 \\
\hline $40-49$ & 10 & 20 & 5 & 20 & 5 & 20 \\
\hline $50-59$ & 6 & 12 & 4 & 16 & 2 & 8 \\
\hline $60-69$ & 4 & 8 & 2 & 8 & 2 & 8 \\
\hline $70-79$ & 1 & 2 & 0 & 0 & 1 & 4 \\
\hline $80-89$ & 2 & 4 & 1 & 4 & 1 & 4 \\
\hline Drug Resistance & 9 & 18 & 7 & 28 & 2 & 8 \\
\hline INH & 6 & 12 & 5 & 20 & 1 & 4 \\
\hline Multi-drug & 3 & 6 & 2 & 8 & 1 & 4 \\
\hline \multicolumn{7}{|l|}{ Treatment Sites } \\
\hline PMD & 28 & 56 & 8 & 32 & 20 & 80 \\
\hline TB Clinic & 19 & 38 & 16 & 64 & 3 & 12 \\
\hline County Clinic & 3 & 6 & 1 & 4 & 2 & 8 \\
\hline
\end{tabular}


Table 2

Completion of Therapy Rate and Relapse Rate

\begin{tabular}{|c|c|c|c|c|c|c|}
\hline & \multicolumn{2}{|c|}{$\begin{array}{l}\text { Combined Group } \\
\qquad(N=50)\end{array}$} & \multicolumn{2}{|c|}{$\begin{array}{l}\text { DOT Group } \\
\qquad(n=25)\end{array}$} & \multicolumn{2}{|c|}{$\begin{array}{l}\text { Non-DOT Group } \\
\qquad(n=25)\end{array}$} \\
\hline & Frequency & Percent & Frequency & Percent & Frequency & Percent \\
\hline \multicolumn{7}{|l|}{ Complete to } \\
\hline Treatment & 46 & 92 & 25 & 100 & 21 & 84 \\
\hline Total Relapse & 2 & 4 & 0 & 0 & 2 & 8 \\
\hline \multicolumn{7}{|l|}{ Drug-Resistant } \\
\hline Relapse & 1 & 2 & 0 & 0 & 1 & 4 \\
\hline \multicolumn{7}{|l|}{ Non-Drug } \\
\hline Resist. Relapse & 1 & 2 & 0 & 0 & 1 & 4 \\
\hline
\end{tabular}

Table 3

Sputum Conversion Rate - Weeks

\begin{tabular}{llllc}
\hline & R & Range & Mean & Standard \\
& & & Deviation \\
\hline Non-DOT Group & 21.0 & 4 to 16 & 10.29 & 2.80 \\
\hline DOT Group & 25.0 & 2 to 7 & 4.72 & 1.77 \\
\hline
\end{tabular}

